\title{
IMPLICAÇÕES ESTRATÉGICAS DA RESPONSABILIDADE SOCIAL CORPORATIVA
}

\author{
Simone Ruchdi Barakat \\ Doutoranda em Administração \\ Faculdade de Economia, Administração e Contabilidade - FEAC \\ Universidade São Paulo \\ simonebarakat@usp.br \\ Edison Fernandes Polo \\ Livre-docente em Administração \\ Professor Associado da Faculdade de Economia e Administração \\ Universidade São Paulo - USP \\ polo@usp.br
}

\begin{abstract}
RESUMO
Apesar da crescente evidência da Responsabilidade Social Corporativa (RSC), ainda não é possível identificar uma definição comum ou um conjunto de princípios centrais sobre o assunto. Pesquisadores ainda discutem o significado de ser socialmente responsável e os impactos do desempenho social no desempenho financeiro. Diante dessa discussão, este estudo teve como objetivo analisar as implicações estratégicas da RSC por meio de uma revisão sistemática da literatura. Foi proposto um modelo teórico em que a RSC leva à geração de valor para empresa, impactando positivamente tanto no desempenho social quanto no desempenho financeiro. Alguns desafios foram mostrados para evidenciar que utilizar a RSC para gerar vantagem competitiva exige considerações a respeito da natureza dos recursos que a empresa possui, da relação das atividades de RSC com a atividade principal da empresa, da disposição dos consumidores em pagar um preço prêmio por características e atributos de RSC e da capacidade da empresa em gerar valor reputacional e inovativo por meio da RSC.
\end{abstract}

Palavras-chave: Estratégia; Geração de valor; Responsabilidade social corporativa; Resource based view; Valor compartilhado.

\section{STRATEGIC IMPLICATIONS OF CORPORATE SOCIAL RESPONSIBILITY}

\begin{abstract}
Despite the growing evidence of Corporate Social Responsibility (CSR), it is not possible to find a common definition or a set of core principles on the topic. Researchers discuss the meaning of being socially responsible and the impacts of social performance on financial performance. Thus, this study aimed to analyze the strategic implications of CSR using a systematic review of the literature. We proposed a theoretical model describing how CSR creates value to business, which can impact positively social performance and financial performance. The use of CSR to generate competitive advantage requires considerations about the nature of the company's resources, the relationship between CSR activities and the company's main activity, the willingness of consumers to pay a premium price for characteristics and attributes of CSR and the company's ability to generate innovative and reputational value with CSR.
\end{abstract}

Key words: Strategy; Value creation; Corporate social responsibility; Resource based view; Shared value. 


\section{INTRODUÇÃO}

Poucos assuntos na área de Administração de Empresas despertam tanta controvérsia e contestação como a Responsabilidade Social Corporativa (RSC). Esse campo de estudo está em crescente desenvolvimento, porém ainda não é possível evidenciar um maior refinamento e operacionalização dos conceitos gerais centrais (DeBakker, Groenwegen, \& Den Hond, 2005; Moretti \& Campanario, 2009). O conhecimento sobre RSC pode ser descrito como "em estado contínuo de emergência" (Lockett, Moon, \& Visser, 2006). É possível encontrar tantas definições para o termo que até o presente não existe um consenso para o seu significado (McWilliams, Siegel, \& Wright, 2006). Um extenso levantamento dessas visões foi realizado por Carroll (2008), o qual mostrou uma grande variedade de pressupostos sobre o que deve abanger a responsabilidade da empresa. Nos extremos dessas visões, encontra-se, por um lado, Milton Friedman (1970), que afirma que a única responsabilidade social da empresa é aumentar seus lucros, enquanto, por outro lado, Keith Davis (1973) argumenta que a RSC requer considerações sobre questões que vão além dos requisitos econômicos, técnicos e legais da firma.

No entanto, a principal discussão, que tem dominado a pesquisa no campo nos últimos 30 anos, refere-se aos impactos financeiros da RSC (Crane, McWilliams, Matten, Moon, \& Siegel, 2008; Aguinis \& Glavas, 2012). Diversos trabalhos foram realizados com o objetivo de comparar o desempenho social com o desempenho financeiro das empresas, porém os resultados encontrados são ambíguos (Husted, Allen, \& Kock, 2015). Enquanto algumas pesquisas encontraram desempenho superior para empresas consideradas mais responsáveis, outras não encontraram diferenças e, ainda, outras encontraram desempenho inferior (Crane et al., 2008; McWilliams \& Siegel, 2011). A inconsistência desses achados pode estar relacionada à inconsistência na própria definição de RSC e nas diferentes formas usadas para medir o desempenho empresarial.

Diante dessa discussão, diversos autores (Hart, 1995, 1997; Porter \& Kramer, 2002, 2006; McWilliams \& Siegel 2001, 2011; McWilliams et al., 2006; Husted \& Salazar, 2006; Boesso, Favotto, \& Michelon, 2015) têm defendido que a RSC somente será boa para os negócios se for exercida de forma estratégica. A utilização das ações de RSC no planejamento estratégico pode ser fonte de oportunidades, inovações e vantagem competitiva (Hamza \& Dalmarco, 2012).

Para elucidar essa questão, este estudo teve como objetivo analisar as implicações estratégicas da RSC. Para isso, foi feita uma revisão sistemática da literatura sobre RSC Estratégica, seguida de uma análise desse conceito à luz da Resource Based View (RBV). Em seguida, foi proposto um framework em que a RSC Estratégica gera valor para empresa, impactando positivamente no desempenho social e no desempenho financeiro. Finalmente, foram levantados os desafios para utilização da RSC Estratégica.

\section{FUNDAMENTAÇÃO TEÓRICA}

Por se tratar de um trabalho teórico, a ferramenta de pesquisa empregada foi a revisão sistemática da literatura. Esse tipo de pesquisa utiliza, como fonte de dados, a literatura acadêmica sobre determinado tema, permitindo que o processo de escolha dos estudos seja sistemático e com base em critérios de relevância (Sampaio \& Mancini, 2007). A vantagem do processo de revisão sistemática em relação à revisão narrativa é possibilitar uma busca exaustiva do que foi publicado sobre o tema e evitar um possível viés do pesquisador ao selecionar os estudos. Nesse tipo de pesquisa, a estratégia de busca deve ser reportada para garantir a replicabilidade e transparência do processo (Tranfield, Denyer, \& Smart, 2003).

A revisão dessa pesquisa foi realizada na base de dados Ebsco - Business Source Complete e ISI -Web of Knowledge. Os termos de busca utilizados foram: Corporate Social Responsibility e Strategy (simultaneamente) e Strategic Corporate Social Responsibility. O período de publicação dos estudos não teve delimitação, permitindo a coleta de artigos publicados a qualquer momento. 
Os resultados da revisão são mostrados por meio de análise descritiva e "análise temática". A análise descritiva mostra o panorama das pesquisas, os grandes temas abordados e marcos evolutivos. A "análise temática" é feita por meio de uma abordagem agregativa e interpretativa, que ressalta o que já é conhecido e estabelecido sobre o tema, identifica principais contribuições dos trabalhos, relaciona-os entre si, sempre que possível, e identifica temas-chaves e problemas de pesquisa emergentes (Tranfield et al., 2003).

A partir das análises feitas por meio da revisão sistemática na literatura sobre RSC, é possível distinguir quatro grupos principais de fundamentações teóricas: 1) Desempenho Social Corporativo ou Corporate Social Performance (CSP), baseado na sociologia; 2) Teoria do Valor ao Acionista, que veio de uma teoria particular da economia, chamada por alguns autores de "fundamentalismo"; 3) Teoria dos Stakeholders, baseada em perspectivas éticas; e 4) Cidadania Corporativa, cujas raízes estão em estudos políticos (Garriga \& Melé, 2004). A classificação das fundamentações teóricas da RSC foi realizada considerando-se o foco principal de cada uma, porém em muitos casos essa tarefa não é tão simples, uma vez que abordam mais de um aspecto e existem conexões entre elas.

A Figura 1 mostra o resumo das principais fundamentações teóricas mapeadas de RSC, suas origens, ideias principais e principais autores. Em seguida, cada uma dessas correntes teóricas é explicada.

\begin{tabular}{|c|c|c|c|}
\hline Teoria de RSC & Origem & Ideia Principal & Principais Autores \\
\hline $\begin{array}{l}\text { Desempenho Social } \\
\text { Corporativo (CSP) }\end{array}$ & Sociologia & $\begin{array}{l}\text { "Assumir responsabilidades sociais não } \\
\text { é uma questão primeiramente econômica, } \\
\text { mas sim uma questão social e ética". }\end{array}$ & $\begin{array}{l}\text { Davis (1973); Wood } \\
\text { (1991). }\end{array}$ \\
\hline $\begin{array}{l}\text { Teoria do valor ao } \\
\text { acionista }\end{array}$ & Economia & $\begin{array}{l}\text { "A única responsabilidade dos negócios } \\
\text { é gerar lucros aos seus acionistas". }\end{array}$ & $\begin{array}{c}\text { Friedman (1970), } \\
\text { Lazonick e O’Sullivan } \\
\text { (2000). }\end{array}$ \\
\hline Teoria dos Stakeholders & Ética & $\begin{array}{l}\text { "A empresa é gerida com a finalidade } \\
\text { de gerar benefícios para seus clientes, } \\
\text { fornecedores, proprietários, funcionários } \\
\text { e comunidades locais, além de manter } \\
\text { sua sobrevivência". }\end{array}$ & $\begin{array}{l}\text { Jones (1980); } \\
\text { Clarkson (1995). }\end{array}$ \\
\hline Cidadania Corporativa & $\begin{array}{l}\text { Ciências } \\
\text { Políticas }\end{array}$ & $\begin{array}{l}\text { "A empresa, como parte da sociedade, } \\
\text { deve agir como uma "boa cidadã". }\end{array}$ & $\begin{array}{l}\text { Wood e Logsdon, } \\
\text { (2002); Matten e Crane } \\
\text { (2005). }\end{array}$ \\
\hline
\end{tabular}

Figura 1. Principais Teorias da Responsabilidade Social Corporativa

Fonte: Elaborada pelos autores

\subsection{Desempenho Social corporativo (CSP)}

O Desempenho Social Corporativo ou Corporate Social Performance (CSP) é entendido como a configuração, na organização empresarial, de princípios de responsabilidade social, processos de respostas a exigências sociais e políticas, programas e resultados tangíveis, que refletem a relação da empresa com a sociedade (Wood, 1991). A CSP sustenta que as empresas, além das responsabilidades econômicas e legais, também possuem responsabilidades com os problemas sociais causados pelos negócios ou por outros motivos. Isso inclui exigências éticas e ações discricionárias ou filantrópicas realizadas pelas empresas em favor da sociedade. Em outras palavras, melhorar o desempenho social significa alterar o comportamento organizacional para produzir menos danos e resultados mais benéficos para a sociedade (Wood, 1991).

A fim de determinar responsabilidades específicas, muitos autores ressaltam a importância de prestar atenção a expectativas da sociedade com relação à empresa e às necessidades sociais. Entre outros argumentos da CSP, é dito que os negócios possuem poder e poder requer responsabilidade. É enfatizado também que a empresa ficaria vulnerável caso seu desempenho seja contrária às expectativas das pessoas que constituem o ambiente social da empresa (Davis, 1975). A

Revista de Gestão Social e Ambiental - RGSA, São Paulo, v. 10, n. 2, p. 37-52, maio/ago. 2016. 
reputação da empresa é relacionada também com a aceitação da comunidade na qual ela opera. Não obstante as consequências econômicas no longo prazo para a empresa, as quais nem sempre são fáceis de medir, não seja a principal consideração para muitos autores, que apontam que assumir responsabilidades sociais não é considerado primeiramente uma questão econômica, mas uma questão social e ética: ser responsável é fazer a coisa certa (Garriga \& Melé, 2004).

\subsection{Teoria do valor ao acionista}

A teoria do Valor ao Acionista afirma que a única responsabilidade social dos negócios é gerar lucros e seu principal objetivo é aumentar o valor econômico da empresa para seus acionistas. A empresa somente poderia se engajar em outras atividades sociais caso fosse exigida por lei ou se contribuísse para maximização do valor ao acionista. Essa teoria está fundamentada na teoria econômica neoclássica, a qual está primeiramente preocupada com a maximização da utilidade do acionista. O principal representante dessa visão é Milton Friedman que, em seu famoso artigo publicado na New York Times Magazine em 1970, afirmou que a única responsabilidade da empresa perante a sociedade é a maximização dos lucros aos acionistas, dentro do quadro legal e dos costumes éticos do país (Friedman, 1970)

$\mathrm{O}$ argumento principal dos que defendem essa corrente, é enfatizar a eficiência desse modelo para criar valor. Administrar uma empresa pela maximização do valor ao acionista não consiste somente em aumentar a riqueza dos acionistas, mas também atingir um melhor desempenho econômico de todo o sistema. Essas condições promovem o incentivo à inovação, redução de custos e preços, produtos com valor econômico agregado e capital para futuros investimentos. Além disso, argumentam que o sistema de impostos permite que parte da riqueza gerada seja dividida com a sociedade pela mediação do governo, que, com ações e leis apropriadas, poderia resolver os problemas de desigualdades e outros problemas gerados pelo mercado (Lazonick \& O'Sullivan, 2000).

$\mathrm{Na}$ prática, a maximização do valor ao acionista frequentemente reflete lucros de curto prazo, como redução em despesas com pessoal. Há evidências que o sucesso econômico no longo prazo não pode ser atingido a menos que os gestores considerem não apenas os interesses dos acionistas, mas também os interesses dos funcionários, clientes, fornecedores, comunidades locais e outros grupos ligados às atividades da empresa- os chamados stakeholders (Clarkson, 1995).

\subsection{Teoria dos stakeholders}

Ao contrário da Teoria dos Acionistas, a Teoria dos Stakeholders leva em consideração os indivíduos ou grupos que possuem ligação com a empresa. A definição de stakeholder mais conhecida e utilizada foi criada por Freeman (1984, p. 46), que afirma que stakeholder significa "[...]qualquer grupo ou indivíduo que pode afetar ou é afetado pelo alcance dos propósitos da empresa" Nessa visão, a empresa é gerida com a finalidade de propiciar benefícios para seus clientes, fornecedores, proprietários, funcionários e comunidades locais, além de manter a sobrevivência da empresa (Jones, 1980). Essa teoria pode ser considerada como uma teoria de RSC, uma vez que fornece um framewok normativo para responsabilidade da empresa perante a sociedade.

Essa teoria pode ser considerada eticamente superior à teoria do valor ao acionista por considerar os diretos e interesses legítimos dos stakeholders, e não apenas o que é requerido por lei. Além disso, contribui para um conceito menos vago de RSC, ao abordar os interesses e as práticas concretas e, ao visualizar responsabilidades, a grupos específicos afetados pelas atividades das empresas (Clarkson, 1995). Outro ponto forte da teoria, é que não é somente uma teoria ética, desconectada da administração dos negócios, mas uma teoria relacionada ao sucesso dos negócios.

Alguns críticos dessa teoria afirmam que ela não fornece funções específicas e objetivas para as empresas, uma vez que balancear os interesses dos stakeholders abandona uma base 
objetiva para avaliar as ações dos negócios. Além disso, a teoria permite várias interpretações e cada interpretação nos fornece um diferente grupo de stakeholders e salienta a importância de diferentes valores, direitos e interesses. Assim, interpretações de diferentes stakeholders levariam a diferentes distribuições de benefícios e deveres (Garriga \& Melé, 2004).

\subsection{Teoria da cidadania corporativa}

O termo cidadania vem da ciência política e contém a ideia geral de fazer parte de uma comunidade. Dessa forma, a teoria da cidadania corporativa considera que a empresa seja parte da sociedade e, por essa razão, deve contribuir com o bem comum, principalmente, na comunidade onde atua, como "boa cidadã". O conceito central de cidadania é participação ao invés de direitos individuais. Essa teoria está mais focada em deveres, responsabilidades e possíveis parcerias das empresas com grupos da sociedade e instituições (Matten \& Crane, 2005).

$\mathrm{Na}$ década de 1990, o conceito de cidadania corporativa ganhou força e atraiu a atenção de empresas devido, principalmente, ao processo de globalização e ao grande poder das empresas multinacionais, uma vez que o conceito de cidadania corporativa possui um apelo global. No entanto, não existe ainda uma teoria completa sobre Cidadania Corporativa, apesar de os trabalhos de alguns pesquisadores (Logsdon \& Wood, 2002; Matten \& Crane, 2005) tentarem desenvolver conceitos e teorias normativas.

As principais críticas a essa teoria referem-se principalmente ao conceito em si, que é muito difuso e pode incluir diversos tópicos, tais como parceria público-privada, práticas corporativas éticas, contribuições corporativas, voluntariado corporativo e gestão corporativa de marca, imagem e reputação. Outra crítica a essa abordagem é que não agrega nada novo para o entendimento da relação entre empresa e a sociedade (Garriga \& Melé, 2004).

\section{DISCUSSÃO}

A classificação apresentada é útil para a contextualização dos diversos estudos existentes sobre RSC, os quais utilizam diversos conceitos e fontes para tratar da mesma questão. Essa classificação considera o foco principal de cada uma das fundamentações teóricas, porém, na maioria dos casos, elas abordam mais de um aspecto e há muitas conexões entre elas. Este trabalho busca conciliar essas teorias, mostrando que o desempenho social pode também gerar desempenho financeiro.

\subsection{Responsabilidade Social Corporativa Estratégica}

O uso da RSC com o objetivo de gerar valor para empresa foi chamado de RSC Estratégica pela primeira vez por Baron (2001). Esse autor defende que, caso a motivação da empresa seja servir à sociedade, mesmo em detrimento dos lucros, então a ação deve ser considerada socialmente responsável, mas caso a motivação seja servir aos lucros, então a ação deve ser considerada privadamente responsável. Para essas últimas ações, poderá haver benefícios sociais que excedam o custo das ações das firmas, porém não mudam a motivação. Por exemplo, disponibilizar creche pode diminuir o número de crimes realizados por jovens, mas a empresa pode oferecer a creche somente porque aumenta a frequência dos trabalhadores e diminui os custos com absenteísmo. No entanto, para McWilliams e Siegel (2011), apesar de essa distinção ser razoável, não é viável, pois não seria possível determinar a real motivação dos gestores. A partir desse pressuposto, McWilliams e Siegel (2011, p. 1481) consideram que RSC Estratégica seja "[...]qualquer atividade responsável que permita à empresa atingir vantagem competitiva sustentável, independentemente da motivação dos gestores".

A lógica da utilização da RSC para gerar tanto benefícios para sociedade quanto para empresa baseia-se na existência de uma interdependência entre ambas. Essa interdependência 
assume duas formas. A primeira é de dentro para fora, em que a empresa afeta a sociedade por meio de suas atividades na cadeia de valor. A segunda é de fora para dentro, em que as condições externas exercem influência sobre as empresas, é o chamado contexto competitivo (Porter \& Kramer, 2006).

O contexto competitivo sempre foi importante para a estratégia, pois variáveis contextuais, tais como disponibilidade de funcionários capacitados e motivados; eficiência da infraestrutura local; tamanho e sofisticação do mercado local; e escopo da regulamentação governamental, afetam a capacidade competitiva da empresa. Ao analisar os elementos do contexto competitivo, uma empresa pode identificar as áreas em que o valor social coincide com o econômico e que mais irão beneficiar a sua própria competitividade, buscando o benefício social combinado com o benefício financeiro (Porter \& Kramer, 2002).

$\mathrm{Na}$ RSC Estratégica, a empresa deve se concentrar em questões que tenham alguma interseção com sua área de atuação e, em geral, quanto maior o vínculo de uma questão social com a atividade da empresa, maior a oportunidade de alavancar recursos em benefício da empresa e da sociedade. Iniciativas de RSC relevantes para estratégia têm maior probabilidade de gerar impactos positivos no desempenho financeiro corporativo, assim como iniciativas relacionadas às preferências dos grupos de stakeholders mais salientes ou mais importantes para a empresa (Boesso et al., 2015).

De acordo com a lógica dos stakeholders, a RSC engloba um conjunto de ações essenciais para a posição competitiva da empresa, que tanto não penaliza o desempenho financeiro como pode gerar valor no longo prazo (Ioannou \& Serafeim, 2015). Contudo, a relação entre desempenho social e desempenho financeiro não ocorre com a simples inclusão de programas de responsabilidade social, mas sim quando é cuidadosamente desenhada para gerar vantagem competitiva para a empresa (Husted et al., 2015). Husted e Allen (2001) criaram um modelo para formular a estratégia de RSC baseado em quatro elementos:

a) Estrutura da indústria: A vantagem competitiva é alcançada pela superioridade em um ou mais dos seguintes fatores competitivos: atributos do produto, imagem do produto, design, preço, serviço ou reputação organizacional. A possibilidade de diferenciação social existe porque há consumidores que fazem decisões de compra baseadas no desempenho social da empresa. A estratégia de diferenciação social é bem-sucedida quando os consumidores estão dispostos a pagar um preço prêmio por produtos sociais e quando são criadas barreiras aos competidores (Reinhardt, 1998).

b) Recursos internos da firma: Esses recursos incluem não somente os tradicionais recursos tangíveis, mas também recursos intangíveis, tais como capital humano, social e reputacional. Esses recursos são desenvolvidos ao longo do tempo, conforme a empresa interage com seus stakeholders. A aplicação desses recursos para oportunidades de mercado e oportunidades sociais representa para cada empresa um posicionamento único e dinâmico (Eisenhardt, 2000).

c) Ideologia e Valores organizacionais: As interpretações dos gestores sobre assuntos sociais e ambientais afetam diretamente a seleção das estratégicas sociais. Um forte comprometimento com responsabilidade social promove um conjunto de valores que não são fáceis de serem imitados (Barney, 1986).

d) Relacionamento com stakeholders: As pessoas e os grupos de pessoas que afetam e são afetados pela empresa formam a estrutura social na qual a empresa opera e determinam por quem ela é responsável. A interação com stakeholders cria temas sociais que promovem oportunidades e ameaças (Clarkson, 1995).

De acordo com esse modelo, a RSC pode ser vista como uma ferramenta estratégia, com a qual a empresa pode gerir tanto ganhos reputacionais, que possibilitam atrair recursos, melhorar o desempenho e construir vantagem competitiva, quanto perdas reputacionais, que resultam da alienação de seus stakeholders. No entanto, as atividades de RSC não geram impacto direto no desempenho financeiro, mas afetam o bottom line por intermédio do estoque de capital reputacional - valor financeiro dos ativos intangíveis (Fombrun, Gardberg, \& Barnett, 2000). 
As atividades geradas por meio do desempenho social e do desempenho financeiro são mutualmente reforçadas. O desempenho financeiro pode prever o desempenho social e vice- versa (Surroca, Tribó, \& Waddock, 2010). Em outras palavras, as empresas podem desenvolver relações com grupos de stakeholders mutualmente benéficas, e empresas socialmente responsáveis podem ser financeiramente recompensadas. Além disso, os efeitos financeiros positivos podem se transformar em mais recursos disponíveis para futuros investimentos em desempenho social (Orlitzky, Schmidt, \& Rynes, 2003).

\subsection{RSC estratégica à luz da Resource Based View (RBV)}

A ideia de que a RSC pode ser usada de forma estratégica pela empresa pode ser analisada por meio da lente da Resourced Based View (RBV). A RBV surgiu de uma frustração com o paradigma estrutura-conduta-desempenho da Organização Industrial, que afirmava que o sucesso da empresa era determinado exclusivamente pelo seu ambiente externo. Os teoristas dessa nova visão consideravam que o paradigma da Organização Industrial era irrealista e limitado, e se voltaram ao trabalho de Penrose (1959) para criar uma nova explicação para o desempenho das empresas. Wernerfelt (1984), Barney (1986), Dierickx e Cool (1989) e Prahalad e Hamel (1990) construíram a RBV em torno das competências internas das empresas. Para essa teoria, a vantagem competitiva está enraizada dentro da empresa. As capacidades ou competências e habilidades gerenciais para reunir esses ativos para produzir desempenho superior determinam a vantagem competitiva (Grant, 1991).

A geração de vantagem competitiva ocorre pela implementação de uma estratégia que agregue valor e gere benefícios para uma empresa, sem que outra concorrente simultaneamente o faça. A vantagem competitiva pode ser atingida por meio dos recursos internos, ou de conjuntos de recursos internos da firma. Para conseguir a vantagem competitiva, os recursos devem ser: 1) valiosos - aqueles que exploram as oportunidades e neutralizam as ameaças do ambiente da firma; 2) raros - aqueles escassos e que não estão presentes em nenhuma empresa concorrente ou potencialmente concorrente; 3) imperfeitamente imitáveis - aqueles que não podem ser imitados; e 4) insubstituíveis - aqueles que não possuem equivalente estratégico (Barney, 1991).

A RBV oferece aos pesquisadores uma ferramenta para refinar a análise de como a política socioambiental da empresa influencia o bottom line por duas razões. Primeiro, a RBV tem um forte foco no desempenho como variável chave. E segundo, da mesma forma que a literatura de RSC, a RBV reconhece explicitamente a importância de conceitos intangíveis, como know-how, cultura corporativa e reputação. Essas complementaridades teóricas oferecem uma oportunidade significativa para destacar a relação entre desempenho social e desempenho financeiro (Russo \& Fouts, 1997).

As atividades de RSC podem ser combinadas com os recursos internos da empresa, de modo que gere valor para a empresa. $\mathrm{O}$ valor é capturado quando consumidores pagam um valor adicional pelos produtos ou serviços da empresa devido a sua participação em projetos relacionados à RSC. O valor pode também ser criado por meio de um novo processo, produto ou serviço mais eficiente que é desenvolvido a partir do envolvimento da empresa em projetos sociais (Husted et al., 2015).

Aplicando as definições de Barney (1991) para gerar vantagens competitivas, as ações de RSC devem ser valiosas, raras, inimitáveis e insubstituíveis. Desta forma, atribuindo estas características às ações e estratégias de RSC, podem-se obter recursos que sejam fonte de vantagens competitivas para a empresa. A RSC contempla diferentes dimensões que podem ser consideradas recursos internos, tais como valores organizacionais, ética nos negócios, relacionamentos com stakeholders, reputação empresarial, entre outros (Pessoa, Nascimento, Neves, \& Oliveira, 2009).

Utilizando a RBV, McWilliams e Siegel (2001) propuseram um modelo de maximização dos lucros para RSC. Nesse modelo, duas companhias produzem produtos idênticos, porém uma delas possui um atributo ou característica social adicionada ao seu produto, o que é, potencialmente, valorizada por alguns consumidores ou por outros stakeholders. A partir disso, os gestores analisam 
o custo-benefício para determinar o nível de recursos que serão alocados para esse atributo ou característica. Eles avaliam a demanda para RSC e o custo para satisfazer essa demanda. Nessa abordagem, a RSC se torna compatível com a visão de Friedman, pois o nível ótimo de resultado social, em cada situação, é calculado para maximizar o valor ao acionista (Husted \& Salazar, 2006).

Essa perspectiva tem diversas implicações estratégicas. Primeiro, a RSC pode ser integrada nas estratégias de diferenciação da empresa, tanto no nível do negócio como no nível da corporação. Dessa forma, deve ser vista como uma forma de investimento estratégico. Mesmo quando não é diretamente atrelada a uma característica de um produto ou a um processo de produção, a RSC pode construir ou manter a reputação da empresa. A segunda implicação estratégica dessa perspectiva é a possibilidade de estabelecer previsões de padrões de investimentos em RSC entre empresas e entre indústrias (McWilliams et al., 2006).

O uso da RSC para diferenciação de produto ocorre quando a maioria dos consumidores prefere um produto a outro. Por exemplo, mantendo-se todas as outras características iguais, a maioria dos consumidores prefere um veículo que utiliza menos combustível. No contexto da RSC, isso ocorre quando fica claro na mente do consumidor que o produto com a característica de RSC é melhor do que o produto sem essa característica. Por exemplo, a versão híbrida do Accord Honda gera menos poluição que o Accord Honda padrão, então é claro para maioria dos consumidores que o modelo híbrido é melhor que o modelo padrão, e alguns desses consumidores estarão dispostos a pagar um preço prêmio pelo carro híbrido. Esse tipo de diferenciação pode fortalecer ou manter a reputação da empresa, além de permitir que ela atenda a uma demanda de mercado específica (McWilliams et al., 2006).

\subsection{Proposição do Framework da RSC Estratégica}

A partir da análise realizada sobre as implicações estratégicas da RSC, propõe-se o framework, representado pela Figura 2.

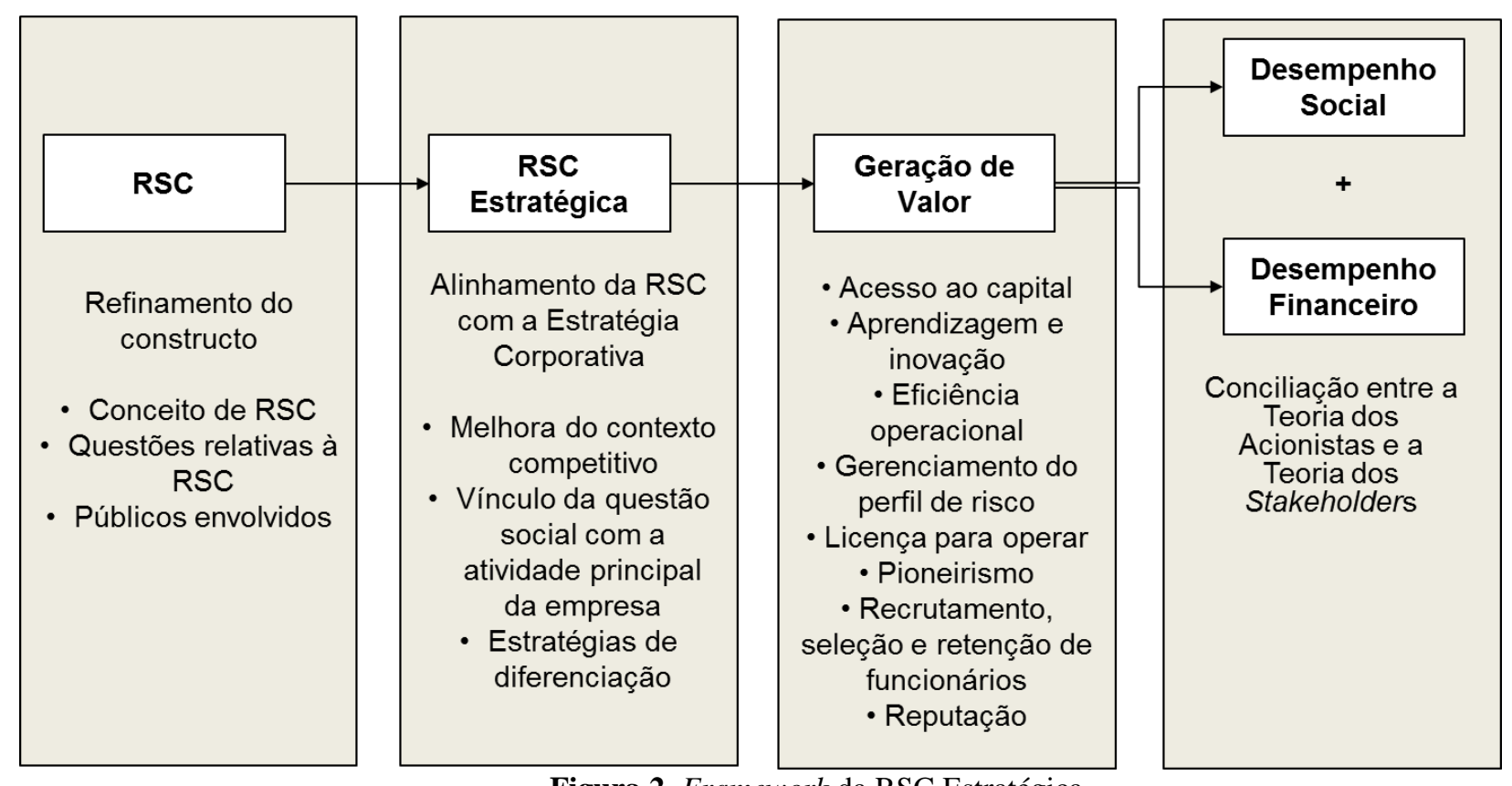

Figura 2. Framework da RSC Estratégica Fonte: Elaborado pelos autores

No framework proposto, a RSC Estratégica inicia-se com a elaboração de definições claras do conceito de RSC para a empresa, quais são as questões relativas a essa responsabilidade e a que públicos ela se dirige. Após essas definições, a empresa é capaz de utilizar a RSC de forma 
estratégica, por meio da melhora do contexto competitivo, da busca de questões sociais que tenham vínculo com sua atividade principal e de estratégias de diferenciação. Com isso, é possível a geração de valor, por meio de benefícios explicados a seguir, gerando tanto desempenho social quanto financeiro.

\subsubsection{Acesso ao capital}

Empresas consideradas responsáveis têm acesso mais rápido e barato ao crédito (Marcondes \& Bacarji, 2010). Em um estudo empírico, Cheng, Ioannou e Serafeim (2014) descobriram que empresas com maior desempenho em RSC possuem menores restrições de acesso ao financiamento. Outra evidência é a criação de diversos fundos de investimentos com base em critérios de RSC, os chamados Socially Responsible Investment (SRI) (BM\&FBovespa, 2012). A demanda por esses fundos cresceu exponencialmente nos últimos anos. No Brasil, o valor dos ativos de SRI cresceu 55,34\% de 2006 a 2012 e, nos Estados Unidos, esse valor aumentou 380\% de 1995 a 2010 (BM\&FBovespa, 2012).

\subsubsection{Aprendizagem e inovação}

A RSC pode gerar criatividade e inovação. A abertura da empresa para relações que vão além dos negócios possibilita novas percepções sobre atividades, processos e desenvolvimento de novos produtos (Molteni, 2006; Hart \& Dowell, 2011). Muitas organizações atuam com parceiros para identificar novas abordagens que proporcionam benefícios para a empresa e, ao mesmo tempo, combatem um problema social ou ambiental (Porter \& Kramer, 2006).

\subsubsection{Eficiência operacional}

A eficiência operacional significa exercer atividades semelhantes melhor do que seus competidores, incluindo todo tipo de prática que permite a empresa utilizar, da melhor forma, os seus recursos (Porter, 1996). Atividades de RSC podem, por exemplo, reduzir desperdícios e reciclar materiais descartáveis, gerando redução de custos para as empresas, além de diminuir a poluição do meio ambiente. Os custos gerados pelos sistemas de proteção ambiental podem ser menores que os custos do uso inadequado dos recursos (Hart, 1995; Porter \& Van der Linde, 1995). Portanto, as empresas podem ganhar vantagem de custo por meio da utilização de estratégias ambientais, pois aproveitam de forma mais eficiente os seus recursos e ativos (Christmann, 2000).

\subsubsection{Gerenciamento do perfil de risco}

A percepção do risco das empresas tem sido ampliada e passou a compreender riscos de longo prazo relativos a questões sociais e ambientais. As demandas dos stakeholders podem ser uma potencial ameaça à viabilidade das atividades da organização. A forma como as empresas respondem a essas demandas pode evitar, por exemplo, boicotes de consumidores, ações judiciais de responsabilidade e aumento de passivo trabalhista (Kurucz, 2008). Uma pesquisa conduzida pela Taylor Nelson Soffres com 302 analistas financeiros e gerentes de fundos na Europa encontrou que $86 \%$ deles acreditam que o gerenciamento do risco social e ambiental terá um impacto positivo significativo no valor de mercado da empresa no longo prazo (Arthur D. Little, 2003).

\subsubsection{Licença para operar}

Manter uma política eficiente de RSC proporciona às empresas uma melhor relação com seus stakeholders e com a opinião pública em geral. Empresas com problemas nessa área podem ser 
alvos constantes de críticas e passam a ter sua forma de atuação com relação a diversos assuntos sociais e ambientais questionada por consumidores, ONGs e ativistas (Kurucz, 2008).

\subsubsection{Pioneirismo}

O pioneirismo está relacionado com a crença que as empresas que atuam. além de suas obrigações legais com relação a questões de responsabilidade social e ambiental se beneficiarão no longo prazo, pois ocuparão uma posição privilegiada em um cenário em que as atividades estarão mais regulamentadas (Madariaga \& Cremades, 2010).

\subsubsection{Recrutamento, motivação e retenção de funcionários}

A reputação da empresa como responsável afeta sua reputação como boa empregadora. A empresa que conta com uma política de RSC bem ajustada tem maiores chances de ter um bom clima organizacional (Branco \& Rodrigues, 2006) e recrutar e reter os melhores talentos (Fombrun et al., 2000; Barrena-Martínez, López-Fernández, Márquez-Moreno, \& Romero-Fernández, 2015). Estudos, tais como de Backhaus, Stone e Heiner (2002), que exploraram a relação entre desempenho social e atratividade de funcionários, chegaram à conclusão que pessoas que procuram emprego consideram alguns fatores, tais como relações com a comunidade, relação com trabalhadores e questões relativas a produtos para escolherem as empresas nas quais trabalhar.

\subsubsection{Reputação}

A reputação faz parte dos ativos intangíveis da empresa e é construída e mantida pela capacidade da empresa em atender as expectativas dos múltiplos stakeholders (Fombrun et al., 2000). De acordo com um relatório realizado pela empresa de consultoria Arthur D. Little (2003), a proporção do valor da empresa derivada de ativos intangíveis cresceu de $17 \%$ em 1981 para $71 \%$ em 1998. De acordo com esse relatório, estima-se que $96 \%$ do valor da Coca Cola abrangem ativos intangíveis de reputação, conhecimento e marca. Para a Kellog's, os ativos intangíveis representam 97\% de seu valor e para American Express 84\%. Além disso, estudos empíricos, como o de Zyglidopoulos (2001) e Raithel e Schwaiger (2015) demostraram que existe uma relação positiva entre reputação e desempenho financeiro. A reputação pode ser criada ou destruída como consequência da decisão em se engajar ou não em atividades de RSC (Husted \& Allen, 2001; Branco \& Rodrigues, 2006).

\subsection{Desafios para o uso estratégico da RSC}

Muitas vezes, as contribuições sociais das empresas refletem crenças e valores pessoais dos gestores, em vez de estarem vinculadas a objetivos sociais ou empresariais bem formulados (Porter \& Kramer, 2002). Nessas situações, as empresas tratam a RSC como um tema à parte de suas políticas e ações, e não como uma questão a ser considerada no delineamento de suas estratégias (Hamza \& Dalmarco, 2012). As abordagens dominantes à RSC, normalmente, são fragmentadas e desvinculadas da estratégia empresarial, ocultando muitas das grandes oportunidades para que a empresa beneficie a sociedade. Se as perspectivas no campo da RSC forem analisadas com os mesmos arcabouços que norteiam as principais decisões empresariais, ela "[...]pode ser muito mais do que um custo, um entrave ou ação filantrópica, pode ser uma fonte de oportunidades, inovação e vantagem competitiva" (Porter \& Kramer, 2006, p. 54).

No entanto, para que isso ocorra é preciso que haja inovação, mas a maioria das empresas continua a se concentrar em estratégias incrementais, tais como eco eficiência, prevenção da poluição e gestão de produtos (Hart \& Dowell, 2011). Tais estratégias incrementais de RSC não são suficientes para que o desempenho social impacte na geração de valor para a empresa. 
Deve-se ressaltar, também, que uma empresa que se engaja em uma estratégia de RSC, apenas poderá obter retornos acima da média se for capaz de prevenir que seus competidores imitem sua estratégia (Reinherdt, 1998), o que se torna difícil em mercados competitivos, onde as atividades de RSC são transparentes.

Barreiras de entrada podem existir, por exemplo, quando produtos que protejam o meio ambiente são patenteados ou quando processos socialmente responsáveis são baseados em relações de confiança com fornecedores e são raros e difíceis de serem imitados (Barney, 1986). Além disso, a estratégia social normalmente é determinada por uma trajetória e tem uma natureza ambígua, podendo promover oportunidades mais fortes de inimitabilidade (Barney, 1986; Peteraf, 1993). Neste caso, os gestores possuem recursos ou capacidades únicas que lhes permitem empregar estratégias sociais lucrativas, que são difíceis de imitar.

A credibilidade é um recurso particularmente importante para desenvolver uma estratégia de diferenciação social. Sem informações confiáveis, os consumidores dificilmente pagarão um preço prêmio para produtos sociais (Reinhardt, 1998). A credibilidade é um elemento essencial para a reputação organizacional, que só é desenvolvida com esforço consistente no longo prazo e contribui para o desempenho financeiro da empresa (Fombrun \& Shanley, 1990).

O desenvolvimento da reputação para credibilidade está baseado, em parte, na disposição da empresa em fornecer informações sobre seus impactos sociais e ambientais. A credibilidade desenvolvida por algumas empresas é uma capacidade estratégica que foi construída dependentemente da sua trajetória e não pode ser imitada facilmente por seus competidores. Além disso, a consistência da política de credibilidade é realizada ao longo do tempo e entre diversas esferas, dificultando a imitação (Husted \& Allen, 2001).

\section{CONSIDERAÇÕES FINAIS}

As discussões acerca das definições de RSC, suas teorias e suas implicações no desempenho financeiro das empresas continuarão. As questões centrais da RSC, tais como para que serve uma empresa e qual a contribuição desta para a sociedade, são tão antigas quanto os negócios em si. Além disso, o campo do conhecimento que RSC representa é amplo e diverso, abrangendo debates a partir de muitas perspectivas, disciplinas e posições ideológicas. Mesmo em Administração de Empresas, é abordado a partir de muitas subdisciplinas, tais como estratégia, marketing, contabilidade, operações e comportamento organizacional. Isso traz à literatura uma diversidade de perspectivas teóricas, abordagens conceituais e tradições empíricas, que não levam a um consenso sobre conceitos críticos, porém, enriquecem e inspiram o debate.

Este trabalho teve como objetivo analisar as implicações estratégicas da RSC por meio de uma revisão sistemática da literatura. Para isso, foi utilizada a abordagem da RSC Estratégica, que busca conciliar o desempenho social e o desempenho financeiro da empresa, mostrando que é possível gerar valor compartilhado- para a empresa e para a sociedade - por meio da RSC. Com essa abordagem é possível conciliar as visões antagônicas existentes na literatura, entre os defendem que a única responsabilidade dos negócios é gerar lucros aos seus acionistas e os que consideram que as empresas, além das responsabilidades econômicas e legais, possuem responsabilidades com os problemas sociais causados por elas ou por outros motivos.

Este trabalho contribui com a proposição de um framework para análise das implicações estratégicas da RSC que pode ser usado tanto por empresas que desejam estabelecer práticas e políticas de responsabilidade social que impactam positivamente a sociedade e os seus negócios, quanto por pesquisadores da área que buscam entender os mecanismos e as condições que levam à geração de valor por meio da RSC. A suposição de que o alinhamento entre a RSC e a estratégia corporativa leva à geração de valor que, por sua vez, resulta em maior desempenho financeiro e social pode ser validada empiricamente em estudos futuros. 
A proposta do framework possui, também, implicações para os trabalhos que buscam analisar as relações entre desempenho social e financeiro. Recomenda-se que essa relação seja testada considerando-se o papel mediador dos benefícios gerados pela RSC e não de forma direta, como ocorre na maioria dos estudos quantitativos realizados. Estudos futuros podem analisar o impacto da RSC no acesso ao capital, na aprendizagem e inovação, eficiência operacional, no gerenciamento do perfil de risco, na licença para operar, no pioneirismo, recrutamento, na seleção e retenção de funcionários e reputação. E, após essa análise, verificar quais os impactos desses benefícios no desempenho financeiro. Além disso, pode-se verificar se empresas que conseguem estabelecer, de forma mais clara, uma definição de RSC, as questões que envolvem a RSC e os públicos que estão envolvidos nessas questões, conseguem maior vantagem competitiva em relação àquelas que adotam práticas de responsabilidade social de forma não planejada e desalinhada com suas estratégias.

Alguns obstáculos foram mostrados para evidenciar que utilizar a RSC Estratégica para gerar vantagem competitiva às empresas não é trivial e exige considerações a respeito da natureza dos recursos que a empresa possui, da relação das atividades de RSC com a atividade principal da empresa, da disposição dos consumidores em pagar um preço prêmio por características e atributos de RSC e da capacidade da empresa em gerar valor reputacional e inovativo por meio da RSC.

\section{REFERÊNCIAS}

Aguinis, H., \& Glavas, A. (2012) What we know and don't know about corporate social responsibility a review and research agenda. Journal of Management, 38(4), 932-968.

Arthur D. Little. (2003) The business case for corporate responsibility. UK: Beacon Press.

Recuperado em: 2 de fev.2015, de:

http://www.bitc.org.uk/resources/publications/cr_business_case.html.

Backhaus, K.B., Stone, B.A., \& Heiner, K. (2002) Exploring the relationship between corporate social performance and employer attractiveness. Business \& Society, 41(3), 292-318.

Barney, J. (1986) Strategic factor markets: expectations, luck, and business strategy. Management Science, 32(10), 1231-1241.

Barney, J. (1991) Firm resources and sustained competitive advantage. Journal of Management, 17(1), 99-120.

Baron, D. (2001) Private politics, corporate social responsibility and integrated strategy. Journal of Economics and Management Strategy, 10, 7-45.

Barrena-Martínez, J., López-Fernández, M., Márquez-Moreno, C., \& Romero-Fernández, P. M. (2015) Corporate Social Responsibility in the Process of Attracting College Graduates. Corporate Social Responsibility and Environmental Management, 22(6), 408-423.

BM\&FBovespa (2012) Índice de Sustentabilidade Empresarial.2012 Recuperado em: 3 março 2015, de: www.bmfbovespa.com.br/Indices/download/Apresentacao-ISE.pdf.

Boesso, G., Favotto, F., \& Michelon, G. (2015) Stakeholder prioritization, strategic corporate social responsibility and company performance: further evidence. Corporate Social Responsibility and Environmental Management, 22(6), 424-440. 
Branco, M.C., \& Rodrigues, L.L. (2006) Corporate social responsibility and resource-based perspectives. Journal of Business Ethics, 69(2), 111-132.

Carroll, A. B. (2008) A history of corporate social responsibility: concepts and practices. In: Crane, A., McWilliams, A., Matten, D., Moon, J., \& Siegel, D. (2008) The Oxford Handbook of Corporate Social Responsibility. New York: Oxford University Press.

Cheng, B., Ioannou, I., \& Serafeim, G. (2014) Corporate social responsibility and access to finance. Strategic Management Journal, 35(1), 1-23.

Christmann, P. (2000) Effects of "best practices" of environmental management on cost advantage: the role of complementary assets. Academy of Management Journal, 43(4), 663-680.

Clarkson, M. B. (1995) A stakeholder framework for analyzing and evaluating corporate social performance. Academy of Management Review, 92(1), 105-108.

Crane, A., McWilliams, A., Matten, D., Moon, J., \& Siegel, D. (2008) The corporate Social Responsibility Agenda. In: The Oxford Handbook of Corporate Social Responsibiliy. New York: Oxford University Press.

Davis, K. (1973) The case for and against business assumption of social responsibility. Academy of Management Journal, 16(2), 312-322.

Davis, K. (1975) Five propositions for social responsibility. Business Horizons, 18(3), 19-24.

DeBakker, G.A., Groenwegen, P., \& Den Hond, F. (2005) A bibliometric analysis of 30 years of research and theory on corporate social responsibility and corporate social performance. Business and Society, 44(3), 283-317.

Dierickx, I., \& Cool, K. (1989) Asset stock accumulation and sustainability of competitive advantage. Management Science, 35(12), 1504-1511.

Eisenhardt, K. M. (2000) Dynamic capabilities: What are they? Strategic Management Journal, 21, 1105-1121.

Freeman, R. E. (1984) Strategic management: a stakeholder approach. Boston: Pitman.

Fombrun, C., Shanley, C. (1990) What's in a name? Reputation building and corporate strategy. Academy of Management Review, 33(2), 233-258.

Fombrun, C, Gardberg, N. A., \& Barnett, M.L. (2000) Opportunity platforms and safety nets: corporate citizenship and reputational risk. Business and Society Review, 105(1), 85-106.

Garriga, E., \& Melé, D. (2004) Corporate social responsability theories: mapping the territory. Journal of Business Ethics, 53(1-2), 51-71.

Grant, R. M. (1991) The resource-based theory of competitive advantage. California Management Review, 33(3), 114-135. 
Hamza, K. M., \& Dalmarco, D. D. A. S. (2012) Integração entre estratégia competitiva e práticas de responsabilidade social corporativa: um estudo exploratório nos cinco maiores supermercados brasileiros. Revista de Gestão Social e Ambiental, 6(3), 78-95.

Hart, S. L. (1995) A natural-resource-based view of the firm. Academy of Management Review, 20(4), 986-1014.

Hart, S. L. (1997) Beyond greening: strategies for a sustainable world. Harvard Business Review, 75(1), 66-76.

Hart, S. L, \& Dowell, G. A. (2011) Natural-resource-based view of the firm: fifteen years after. Journal of Management, 37(5), 1464-1479.

Husted, B. W., \& Allen, D. B. (2001) Toward a model of corporate social strategy formulation. Paper presented at the Social Issues in Management Division, Academy of Management Conference, August.

Husted, B. W, \& Salazar, J.D.J. (2006) Taking Friedman seriously: maximizing profits and social performance. Journal of Management Studies, 43(1), 75-91.

Husted, B. W., Allen, D. B., \& Kock, N. (2015) Value creation through social strategy. Business \& Society, 54(2), 147-186.

Ioannou, I., \& Serafeim, G. (2015) The impact of corporate social responsibility on investment recommendations: analysts' perceptions and shifting institutional logics. Strategic Management Journal, 36(7), 1053-1081.

Jones, T.M. (1980) Corporate social responsibility revisited, redifined. California Management Review, 22(2), 59-67.

Kurucz, E.C. (2008) The business case for corporate social responsibility. In: Crane, A.; McWilliams, A., Matten, D., Moon, J., \& Siegel, D. The Oxford Handbook of Corporate Social Responsibility. New York: Oxford University Press.

Lazonick, W., \& O’Sullivan, M. (2000) Maximizing shareholder value: a new ideology for corporate governace. Economy and Society, 29(1), 13-35.

Lockett, A., Moon, J., \& Visser, W. (2006) Corporate social responsibility in management research: focus, nature, salience and sources of influence. Journal of Management Studies, 43(1), 115-36.

Logsdon, J. M., \& Wood, D. J. (2002) Business citizenship: from individuals to organizations. Business Ethics Quarterly, 12(2), 155-187.

Madariaga, J.G., \& Cremades, F.R. (2010) Corporate social responsibility and the classical theory of the firm: Are both theories irreconcilable? Innovar Journal, 20(37), 5-19.

Marcondes, A.W., \& Bacarji, C.D. (2010) ISE - Sustentabilidade no Mercado de Capitais. São Paulo: Report Editora, 173p.

Matten, D., \& Crane, A. (2005) Corporate citizenship: towards an extended theoretical conceptualization. Academy of Management Review, 30(1), 166-179. 
McWilliams, A., \& Siegel, D. S. (2001) Corporate social responsibility: a theory of the firm perspective. Academy of Management Review, 26(1), 117-127.

McWilliams, A., \& Siegel, D. S. (2011) Creating and capturing value: strategic corporate social responsibility, resource-based theory, and sustainable competitive advantage. Journal of Management, 37(5), 1480-1495.

McWilliams, A., Siegel, D. S, \& Wright, P.M. (2006) Corporate social responsability: strategic implications. Journal of Management Studies, 43(1), 1-18.

Molteni, M. (2006) The social-competitive innovation pyramid. Corporate Governance, 6(4), 516526.

Moretti, S. L. A., \& Campanario, M. A. (2009) A produção intelectual brasileira em responsabilidade social empresarial - RSE sob a ótica da bibliometria [Edição especial]. Revista de Administração Contemporânea, 13, 68-86.

Orlitzky, M., Schmidt, F. L., \& Rynes, S. L. (2003) Corporate Social and financial performance: a meta-analysis. Organization Studies, 24(3), 403-441.

Pessoa, R.W.A., Nascimento, L.F., Neves, J.A.D., \& Oliveira, G.S. (2009) Estratégia e vantagem competitiva da responsabilidade social empresarial. Gestão \& Regionalidade, 25(74), 79-93.

Peteraf, M.A. (1993) The cornerstones of competitive advantage: a resource-based view. Strategic Management Journal, 14(3), 179-191.

Porter, M. E., \& Kramer, M. R. (2006) Strategy and society: the link between competitive advantage and corporate social responsibility. Harvard Business Review, 84(12), 78-92.

Porter, M. E., \& Kramer, M. R. (2002) The competitive advantage of corporate philanthropy. Harvard Business Review, 80(12), 57-68.

Porter, M.E. (1996). What is strategy? Harvard Business Review, nov-dec, 61-78.

Porter, M. E., \& Linde, V. D. (1995) Toward a new conception of the environmental competitiveness relationship. Journal of Economic Perspectives, 9(4), 97-118.

Prahalad, C. K., \& Hamel, G. (1990) The core competence of the corporation. Harvard Business Review, 68(3), 79-91.

Raithel, S., \& Schwaiger, M. (2015) The effects of corporate reputation perceptions of the general public on shareholder value. Strategic Management Journal, 36(6), 945-956.

Reinhardt, F. L. (1998) Environmental product differentiation: implications for corporate strategy. California Management Review, 49, 43-73.

Russo, M. V., \& Fouts, P. A. (1997) A resource-based perspective on corporate environmental performance and profitability. Academy of Management Journal, 40(3), 534-559. 
Sampaio, R. F., \& Mancini, M. C. (2007) Estudos de revisão sistemática: um guia para síntese criteriosa da evidência científica. Braz. J. Phys. Ther.(Impr.), 11(1), 83-89.

Surroca, J., Tribó, J.A., \& Waddock, S. (2010) Corporate responsibility and financial performance: the role of intangible resources. Strategic Management Journal, 31(5), 463-490.

Tranfield, D., Denyer, D., \& Smart, P. (2003) Towards a methodology for developing evidence-informed management knowledge by means of systematic review. British Journal of Management, 14(3), 207-222.

Wernerfelt, B. (1984) A resource-based view of the firm. Strategic Management Journal, 5(2), 171180 .

Wood, D. J. (1991) Corporate social performance revisited. Academy of Management Review, 16(4), 66-73.

Zyglidopoulos, S.C. (2001) The impact of accidents on firm's reputation for social performance. Business \& Society, 40(4), 416-441.

Data da submissão: 29/08/2015

Data da publicação: 30/04/2016 